\title{
Supraclavicular Neck Mass as Sole Presenting Symptom for Seminoma in an Elderly Male
}

\author{
Justin R. Bond, Michelle Tilley, Sapna Amin, Christopher G. Larsen \\ Department of Otolaryngology, University of Kansas Medical Center, Kansas City, USA \\ Email: jbond@kumc.edu
}

Received April 25, 2013; revised May 26, 2013; accepted June 17, 2013

Copyright (c) 2013 Justin R. Bond et al. This is an open access article distributed under the Creative Commons Attribution License, which permits unrestricted use, distribution, and reproduction in any medium, provided the original work is properly cited.

\begin{abstract}
We report an unusual case of genitourinary malignancy in an otherwise asymptomatic elderly male, which was discovered via workup of a supraclavicular neck mass. We present his clinical workup as well as the pathological workup and how it influenced our decision-making. A review of the literature is also discussed and demonstrates how uncommon it is for seminomas to present in this manner.
\end{abstract}

Keywords: Neck Mass; Supraclavicular; Seminoma; Cervical Metastasis; Lymphadenopathy

\section{Introduction}

Neck mass is a common complaint evaluated in the clinic of the Otolaryngologist. Differential diagnosis for a neck mass is wide and should include infectious, inflammatory, congenital, and certainly neoplastic processes. When neoplasm is diagnosed it is easy to assume upper aerodigestivetract origin given the local and regional lymphatic drainage pathways. However, malignant tumors outside of the upper aerodigestive tract can and do spread to the neck and should always be considered in the differential of a neck mass. We present an interesting case of genitourinary malignancy that was diagnosed through the workup of an asymptomatic neck mass.

\section{Case Report}

A 76-year-old male presented to the Otolaryngology clinic for evaluation of an incidentally discovered left supraclavicular mass. He had a soft carotid bruit detected by his primary care physician two months prior. Carotid ultrasound revealed no stenosis but did show a $4.0 \mathrm{~cm}$ left neck mass laterally displacing the carotid sheath. He is a former smoker with a 116 pack year history. Review of systems is positive for long-term dysphonia from presbylarynges, but is otherwise negative for malignant symptoms. Head and neck examination including fiberoptic exam showed no mucosal lesions of the upper aerodigestive tract. The only positive finding was a left supraclavicular mass just above the clavicular head and medial to the sternocleidomastoid muscle. Further work up commenced with a contrasted CT scan of the neck which demonstrated a $4.0 \times 3.0 \mathrm{~cm}$ soft tissue mass compressing the carotid sheath medially and extending below the clavicle.

Fine needle aspiration (FNA) of the soft tissue mass was performed and was positive for malignant cells. Cytologically, the cells were discohesive and atypical with large oval to round nuclei, prominent nucleoli, and clear cytoplasm. Occasional mitotic figures were identified (Figure 1). Immunohistochemical stains revealed focalvimentin expression, in addition to focal weak positivity for placental-like alkaline phosphatase (PLAP) and CD117. Other epithelial, lymphocytic, neuroendocrine, and melanocytic markers were negative. Due to the paucity of tumor cells remaining in the cell block, the findings were overall inconclusive for a definitive classification of the neoplasm and excisional biopsy was recommended.

A PET scan was obtained preoperatively which was read as positive for hypermetabolic activity in one supraclavicular lymph node and two retroperitoneal lymph nodes, consistent with metastatic lymphadenopathy. No primary site of malignancy was identified.

The patient underwent nasal endoscopy, direct laryngoscopy, and esophagoscopy to more definitively evaluate for a primary lesion of the aerodigestive tract. None was identified so excisional biopsy of the supraclavicular mass was performed. Intra-operative frozen section confirmed the presence of a malignant neoplasm, although definitive classification of the tumor was deferred once 


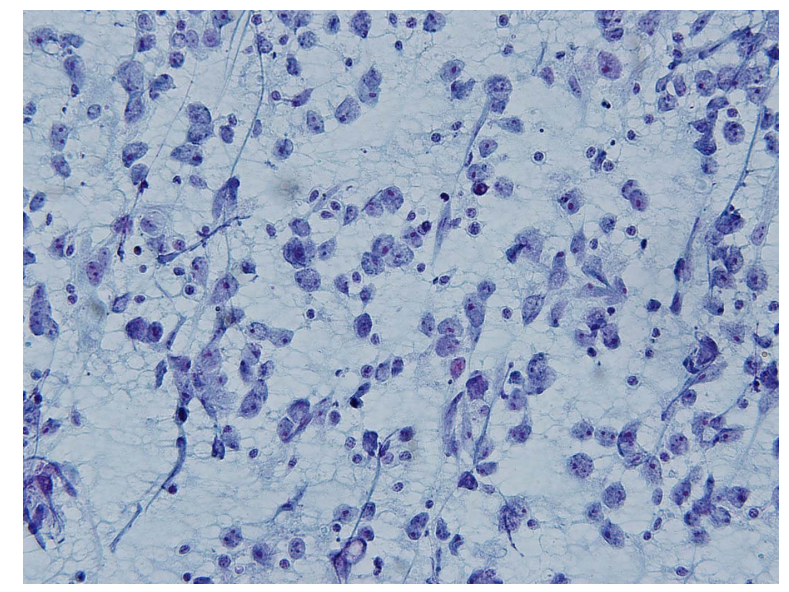

Figure 1. Papanicolaou-stained direct smears exhibited discohesive, atypical cells with oval to round nuclei, prominent nucleoli and clear cytoplasm (Papanicolaou stain; $\times 400$ ).

again.

Final pathologic examination showed a lymph node measuring $5.0 \times 4.5 \times 3.0 \mathrm{~cm}$ with a nodular cut surface. On permanent sectioning, the lymph node showed nodules of metastatic tumor cells divided by intervening fibrous septa with a moderate lymphocytic infiltrate (Figure 2). Examination of these cells on higher power showed loosely cohesive large cells with vesicular nuclei, prominent nucleoli, and clear cytoplasm, cytologically resembling those seen on the previous FNA (Figure 3). Further immunohistochemical stains show the tumor cells to be positive for PLAP and CD117 and focally positive for epithelial markers and CD30 (Figure 4). The cells were negative for human chorionic gonadotropin (HCG), alpha-fetoprotein (AFP), renal cell carcinoma marker, CD3, and CD20. A final diagnosis of metastatic seminoma was rendered.

Given this pathologic diagnosis, a testicular exam was performed and both testes were descended, but no masses were palpated. However, a testicular ultrasound did revealed a $1.5 \mathrm{~cm}$ heterogeneous mass of the left testicle. Serum tumor markers (AFP and beta-HCG) were within normal limits. Oncology consultation was obtained, recommending a chemotherapeutic regimen that consisted of 4 cycles of etoposide and cis-platin. Bleomycin was excluded from this patient's regimen due to his significant smoking history and the risk of pulmonary fibrosis. It was also recommended that he undergo salvage orchiectomy after completion of chemotherapy since penetration to the testicle would not likely occur.

\section{Discussion}

Testicular tumors make up approximately 1\% of cancers in men, and are the most common malignancies of men age 29 - 30 [1]. In general, primary testicular malignancies are divided into seminomas and non-seminomatous

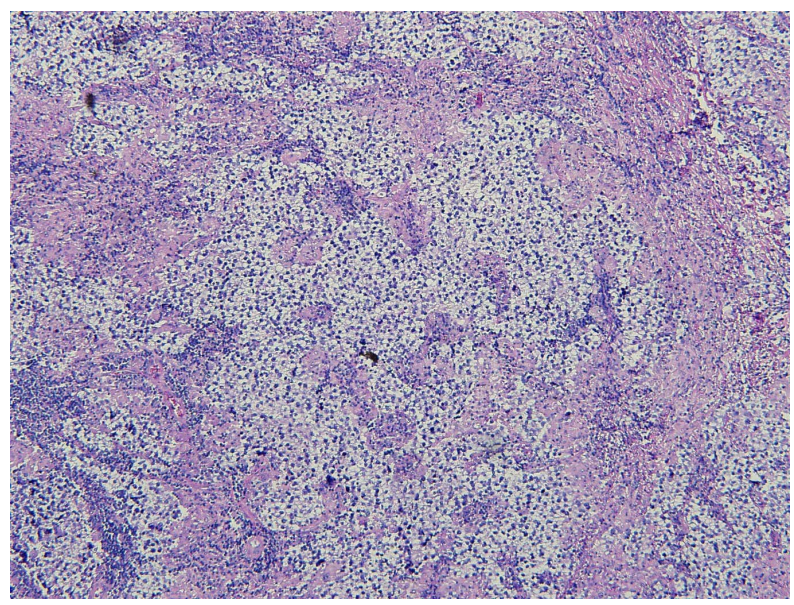

Figure 2. Low-power view of the lymph node with nodules of metastatic tumor cells divided by fibrous septa with lymphocytic infiltrate (Hematoxylin and eosin stain; $\times 100$ ).

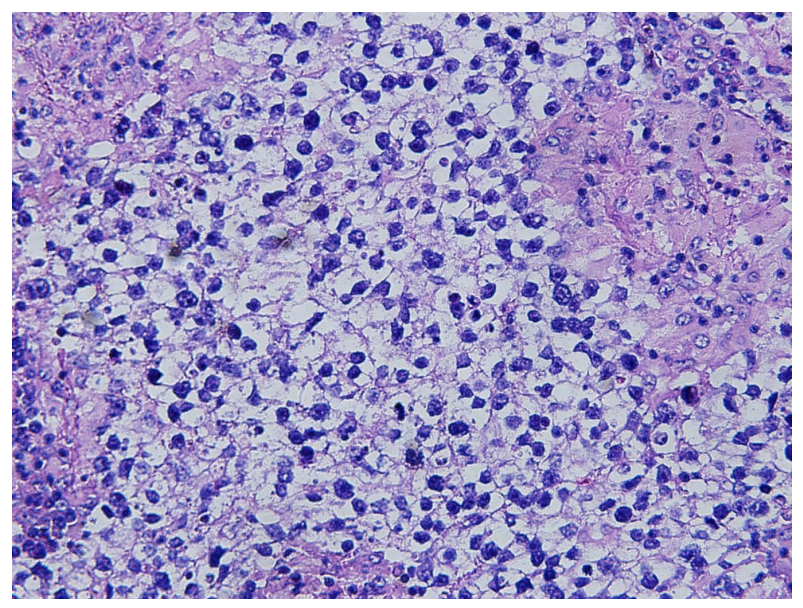

Figure 3. High power view revealed large cells with vesicular nuclei, prominent nucleoli, and clear cytoplasm (Hematoxylin and eosin stain; $\times 400$ ).

germ cell tumors (NSGCTs). vanVledder et al. reviewed 665 patients from January 1997 to June 2009 and found that 492 (76\%) were NSGCTs with the remaining 173 (24\%) representing seminomas [2]. The peak incidence of seminomas is 34 - 45 years [1]. So our patient had an uncommon malignancy at even more uncommon age.

Seminomas typically present as painless testicular enlargement. The classic histological features are large, round to polyhedral cells with clear cytoplasm and a large central nucleus. The tumor cells stain positive for PLAP and do not express AFP or HCG, unlike NSGCTs [1]. The permanent sections and immunohistochemical stains from our patient's specimen were reflective of these classic features.

In a review by Cooper et al. approximately $75 \%$ of seminomas present as stage 1 , with disease limited to the testis [3]. Far less frequent (14\%) is stage 3 disease with metastasis to nonregional lymph nodes or disseminated 


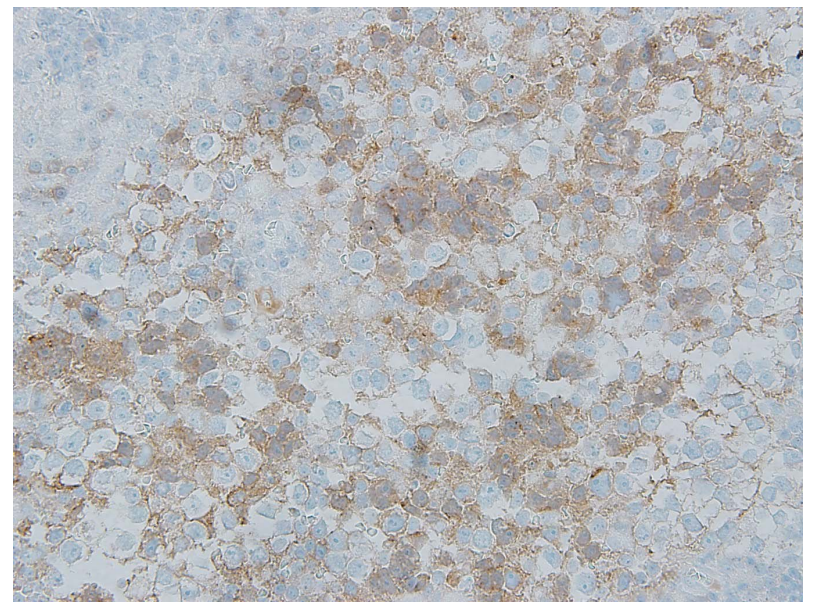

(a)

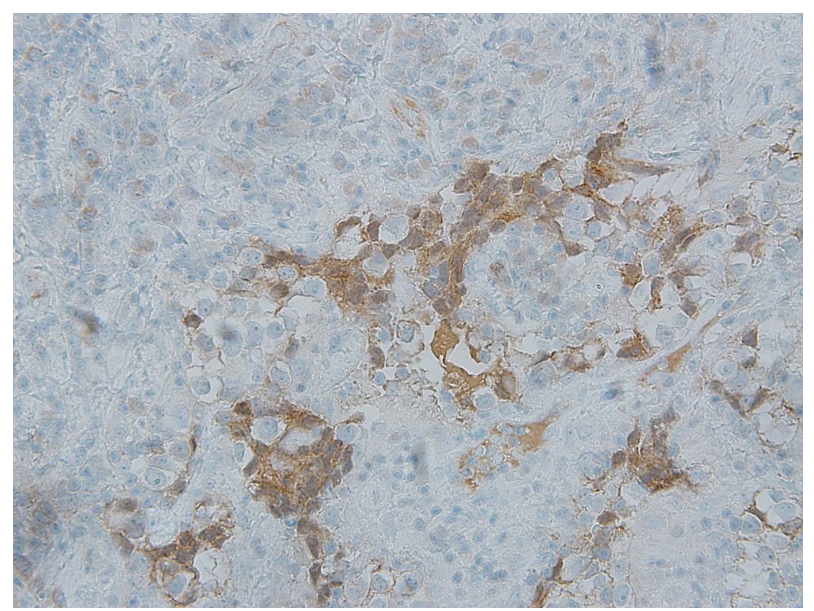

(b)

Figure 4. (a) PLAP immunostain highlights the tumor cells (PLAP immunohistochemical stain; $\times 400$ ); (b) The tumor cells also express CD117 (CD117 immunohistochemical stain; $\times 400)$.

disease [1,3]. All tumors of germ cell origin have the propensity to metastasize via lymphatic pathways. This spread is typically in a sequential pattern, beginning with involvement of abdominal lymph nodes and successive involvement of lymph nodes in the chest and neck [4]. Metastatic tumors can appear in locations that are not in the direct line of spread from the primary site. In the review by van Vledder, $4 \%$ of seminoma patients also had cervical metastasis, with only $5 \%$ of those patients having the neck mass as the initial sign of disease [2]. Wood et al. demonstrated that the cervical metastasis is almost exclusively left sided with 21 of 23 patients having disease in supraclavicular or scalene lymph nodes [4].

Treatment for advanced germ cell tumors includes combination chemotherapy bleomycin-cisplatin-etoposide, followed by surgical salvage for residual disease. Depending of the patient's risk profile, 3 - 4 cycles of chemotherapy are needed [5].

\section{Conclusion}

Case reports of germ cell tumors initially manifesting as a neck mass are rare. It is known that seminomas can and do metastasize to the supraclavicular lymph nodes. However, our patient fit in the exceedingly rare category because he did not present until clinical stage III at an advanced age. Otolaryngologists should remember to include metastatic testicular germ cell tumors in their differential diagnosis of supraclavicular neck mass. These tumors should be included on the differential diagnosis in all age groups because, as we have learned from this case, they may occur at an unexpected age.

\section{REFERENCES}

[1] A. Bahrami, J. Ro and A. Ayala, "An Overview of Testicular Germ Cell Tumors,” Archives of Pathology \& Laboratory Medicine, Vol. 131, No. 8, 2007, pp. 12671280.

[2] M. G. vanVledder, J. A. van der Hage, W. J. Kirkels, et al., "Cervical Lymph Node Dissection for Metastatic Testicular Cancer,” Annals of Surgical Oncology, Vol. 17, No. 6, 2010, pp. 1682-1687. doi:10.1245/s10434-010-1036-X

[3] D. E. Cooper, J. O. L'esperance, M. S. Christman and B. K. Auge, “Testis Cancer: A 20-Year Epidemiological Review of the Experience at a Regional Military Medical Facility,” Journal of Urology, Vol. 180, No. 2, 2008, pp. 577-581.

[4] A. Wood, N. Robson, K. Tung and G. Mead, "Patterns of Supradiaphragmatic Metastases in Testicular Germ Cell Tumours," Clinical Radiology, Vol. 51, No. 4, 1996, pp. 273-276. doi:10.1016/S0009-9260(96)80345-X

[5] A. Flechon, M. Rivoire and J. P. Droz, "Management of Advanced Germ Cell Tumors of the Testis," Nature Clinical Practice Urology, Vol. 5, 2008, pp. 262-276. doi:10.1038/ncpuro1101 